Original Paper http://ajol.info/index.php/ijbcs $\quad$ http://indexmedicus.afro.who.int

\title{
Inventaire, structure morpho-métrique et importance des varans sacrés de Kandi (Nord-Est Bénin)
}

\author{
Ridwane BIO OURE ${ }^{1 *}$, Is-Haquou A DAOUDA ${ }^{1}$, Valentin M. KINDOMIHOU ${ }^{2}$ et \\ Guy Apollinaire MENSAH ${ }^{3}$
}

\author{
${ }^{I}$ Département d'Aménagement et Gestion des Ressources Naturelles FA-Univ. Parakou, Bénin. \\ ${ }^{2}$ Laboratoire d'Ecologie Appliquée, FSA-Univ. Abomey-Calavi, Bénin. \\ ${ }^{3}$ Institut National des Recherches Agricoles du Bénin (INRAB), Bénin. \\ *Auteur correspondant ; E-mail : ridou.bo@gmail.com;02 BP 1406 Parakou,Bénin ; Tél : (00229) 95522808.
}

\section{RESUME}

Les sites naturels sacrés regorgent d'espèces traditionnellement protégées. Cette étude menée de juin 2013 à décembre 2013 à Kandi (milieu soudanien) a permis de décrire les espèces de varans et d'analyser la structure morpho-métrique des varans sacrés. Les observations directes, les enquêtes ethnozoologiques semistructurées sur 74 chefs de ménage et chasseurs et, les mesures corporelles des varans capturés ont signalé la présence de Varanus exanthematicus et Varanus niloticus différenciés à partir de la taille (87\% des enquêtés), forme (61\%), couleur (59\%) et tempérament (52\%). V. niloticus était sacré pour l'ethnie dominante "Batonu". $84 \%$ des captures étaient mâtures, pesaient en moyenne $1484 \mathrm{~g}$ pour $111,3 \mathrm{~cm}$ de long. La longueur moyenne du museau-cloaque était de $44,7 \mathrm{~cm}$ et la queue $65,3 \mathrm{~cm}$. Ils étaient plus courts et moins lourds que $V$. niloticus du Lac Tchad (milieu sahélien) pouvant traduire une adaptation phénotypique. La longueur de la queue (LQ) et du museau-cloaque (LMC) s'ajustent dans le modèle $\mathrm{LQ}=1,19 \mathrm{LMC}+11,74\left(\mathrm{R}^{2}=0,73 ; \mathrm{N}=37\right)$. La longueur totale (L) et la masse corporelle (MC) s'ajustent dans l'équation $\mathrm{MC}=0,03 \mathrm{~L}-1,42 \quad\left(\mathrm{R}^{2}=0,81 ; \mathrm{N}=37\right)$. Toutefois, leur statut écologique doit être analysé en incluant la symbiose hommes-varans.

(C) 2015 International Formulae Group. All rights reserved.

Mots clés: Varans, richesse spécifique, données biométriques, Kandi.

\section{Inventory, morpho-metric structure and importance of sacred monitor lizard of Kandi (Northeastern Benin)}

\begin{abstract}
Sacred natural sites abound in traditionally protected species. This study led from June 2013 to July 2013 at Kandi (Sudanese area) has enabled to describe the species of monitor lizards and to analyze the morpho-metric structure of sacred monitor lizards. Direct observations, semi-structure and ethnozoological surveys on 74 heads of household and hunters, and body measurements of monitor lizards captured have indicated the presence of Varanus exanthematicus and Varanus niloticus differentiated from size (87\% of investigated), form (61\%), color (59\%) and temperament (52\%). V. niloticus was sacred for "batonu" the dominant ethnic. $84 \%$ of the captures were matures, weighed on the average $1484 \mathrm{~g}$ and $111.3 \mathrm{~cm}$ long. The average of snout-vent-length (SVL) was $44.7 \mathrm{~cm}$ and the tail (TL) $65.3 \mathrm{~cm}$. They were more shorts and less
\end{abstract}


heavy than $V$. niloticus of Lake Chad (sahelian environment). It can express phenotype adaptation. Tail length and snout-vent-length fit in the model $\mathrm{TL}=1.19 \mathrm{SVL}+11.74\left(\mathrm{R}^{2}=0.73 ; \mathrm{N}=37\right)$. Total length $(\mathrm{L})$ and body mass (BM) fit in the equation $\mathrm{BM}=0.03 \mathrm{~L}-1.42\left(\mathrm{R}^{2}=0.81 ; \mathrm{N}=37\right)$. Therefore, their status must been analyzed including the symbiosis human-monitor lizards.

(C) 2015 International Formulae Group. All rights reserved.

Keywords: Monitor lizards, specific richness, biometrical data, Kandi.

\section{INTRODUCTION}

En 2009 sur la liste rouge de l'UICN, 293 espèces de reptiles ont été ajoutées dont Varanus mabitang des Philippines et la CITES enregistrait $V$. nebulosus, $V$. komodoensis, V. griseus, $V$. flavescens et $V$. bengalensis dans la catégorie I (Savey, 2009). Les causes sont l'utilisation de stocks importants de varans dans les terraria et en maroquinerie en Europe, Asie et Amérique. En Afrique, des spécimens entiers et des organes de reptiles (crocodiles, serpents, tortues, varans, etc.) entrent dans des recettes alimentaires et médicinales (Bayless et Luisselli, 2001 ; De Buffrénil, 2004 ; Kpéra et al., 2004). Au Bénin, la chasse pour l'alimentation et l'approvisionnement des marchés de cadavres d'animaux, et la perte de l'habitat (Sinsin et al., 2008), favorisent leur disparition. Ce braconnage touche aussi bien les aires non protégées que les aires protégées. Cependant, partout dans le monde dont le Bénin, des peuples tiennent des aires naturelles et des espèces pour sacrées (Kpéra et al., 2004; Mensah et al., 2006 ; Bio Ouré, 2011 ; Djego-Djossou et al., 2012 ; DjègoDjossou et Sinsin, 2009). Dudley et al. (2005) ont trouvé des liens entre des croyances et la conservation. A Sam au Nord-Est du Bénin, des varans ont, du fait de leur caractère sacré, survécu en cohabitant avec les populations. Dans la nature, les varans jouent un rôle biologique (Sandjong Sani et al., 2013a), environnemental (Ciliberti et al., 2011) et écologique comme régulateur des ravageurs de culture. Ils ont colonisé différents types d'habitat et peuvent donc être terrestre, aquatique, saxicole et arboricole (Pianka and King, 2004 ; Schuett et al., 2009 ; Openshaw and Keogh, 2014). En fonction donc du type d'habitat occupé, les varans terrestres auront une forme large, les varans arboricoles auront une forme intermédiaire et les varans saxicoles seront minces (Collar et al., 2011). Les différences d'habitat contribuent donc à la diversité de leur structure morphologique comme les longueurs de la queue et des membres (Aerts et al., 2000; Butler and King 2004; Collar et al., 2010).

$\mathrm{Au}$ Bénin, en dehors des travaux d'inventaire qualitatif des reptiles (Mensah et al., 2006) et sur leur utilisation (Sinsin et al., 2008), les données scientifiques notamment biométriques portant sur les varans sont presque inexistants. Dans ce contexte, cette étude sur les espèces de varans de Kandi, leur morphologie et biométrie permet d'enrichir la base de données des varans du Bénin afin d'une prise de mesure de leur conservation.

\section{MATERIEL ET METHODES \\ Milieu d'étude}

Kandi est située au Nord-Est du Bénin entre $10^{\circ} 90^{\prime}$ et $11^{\circ} 35^{\prime}$ de latitude Nord ; $2^{\circ} 38^{\prime}$ et $3^{\circ} 15^{\prime}$ de longitude Ouest avec 10 arrondissements dont Angaradébou, Sam, Donwari (Figure 1). Son climat est de type soudanien alternant une saison sèche (novembre-mars) et une saison de pluie (avriloctobre). La pluviométrie moyenne de 1981 à 2010 est de $975 \mathrm{~mm}$. La population majoritairement musulmane $(75,45 \%)$, est dominée par le groupe socioculturel Bariba $(31,38 \%)$ (INSAE, 2003). L'économie est dominée par l'agriculture extensive et l'élevage transhumant des bovins. La chasse traditionnelle accentue le braconnage.

\section{Collecte des données}

Les données ont été collectées de juin 2013 à décembre 2013 par des observations directes, mesures corporelles sur les varans sacrés et enquêtes ethnozoologiques semistructurées sur la base de questionnaire (Smart et al., 2005). L'échantillon de 74 personnes est composé de 63 chefs de ménage (33 hommes, 30 femmes) et 20 chasseurs dont 9 font partie des 63 chefs de ménage.

\section{Techniques de capture}

Les captures faites à la main (Luiselli et Akani, 1999) et au fil et dans la matinée (7 h à $10 \mathrm{~h})$ et l'après-midi $(17 \mathrm{~h}$ à $19 \mathrm{~h})$ ont été marquées et numérotées par coloration et amputation des phalanges aux pattes postérieures (Daouda, 2002). Les longueurs du Museau-Cloaque, de la queue, du corps (Figure 2) et la masse (Collar et al., 2011 ; Openshaw et Keogh, 2014) ont été prises. Ils ont été regroupées dans la catégorie d'âge des matures (longueur totale $>85 \mathrm{~cm}$ ) et des immatures (longueur totale $<85 \mathrm{~cm}$ ) (De 
Buffrénil et al., 1994). La catégorisation en subadultes (LMC $<40 \mathrm{~cm}$ ), adultes $(40 \mathrm{~cm}$ $\leq \mathrm{LMC}<50 \mathrm{~cm}$ ) et très adultes $(\mathrm{LMC} \geq 50 \mathrm{~cm})$ (Ciliberti et al., 2011) n'a pas été utilisée du fait du faible taux de capture.

\section{Analyse statistique des données}

$\mathrm{La}$ richesse spécifique et les covariances entre LMC et LQ et, entre la longueur totale (L) et la masse corporelle (MC) ont été établies. Les données ont été traitées au tableur Excel. Le calcul des moyennes, l'estimation des coefficients de variation, la réalisation des figures et les tests de comparaison ont été faits avec le logiciel statistique MINITAB 14.
Le rapport entre la longueur de la queue (LQ) et du museau-cloaque (LMC) a été déterminé pour les varans immatures et les varans matures. $\mathrm{r}=L Q$

$$
\text { LMC }
$$

L'indice de masse corporelle (IMC) aussi bien des varans immatures que matures a été déterminé sur la base de la masse corporelle (MC) et la longueur totale (L).

$$
\mathrm{IMC}=M \mathrm{C} / \mathrm{L}^{2} \quad \text { avec } \mathrm{MC} \text { en }
$$
kilogramme $(\mathrm{kg})$ et $\mathrm{L}$ en mètre $(\mathrm{m})$.
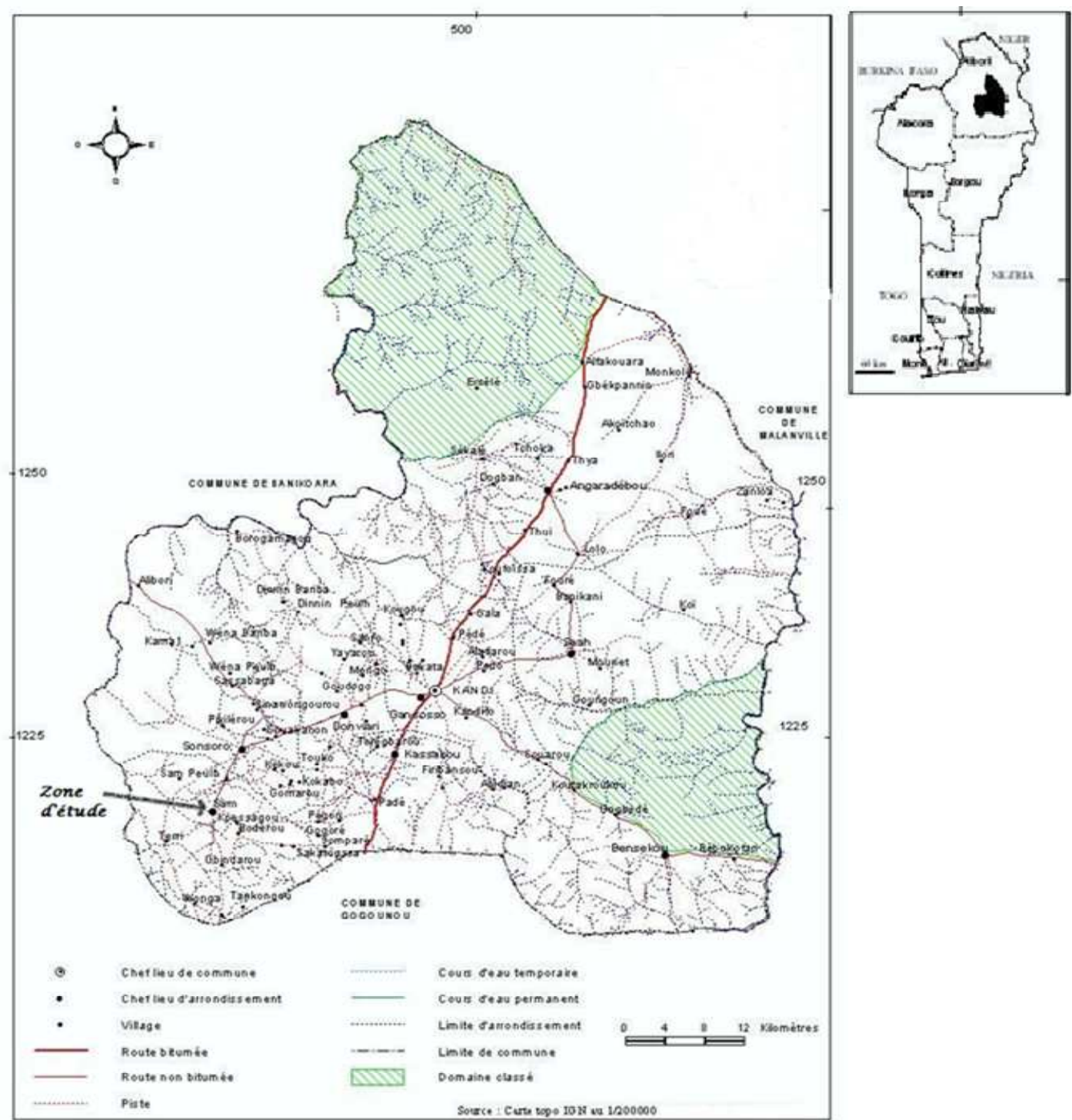

Figure 1 : Situation géographique de Kandi. 


\section{RESULTATS}

Diversité des varans de Kandi

Le varan du Nil (Varanus niloticus) (Figure 3) et le varan de savane (Varanus exanthematicus) ont été les deux espèces signalées à Kandi $(100 \%$ des personnes interrogées) et $V$. niloticus était plus abondante. La population les reconnaît grâce à la différence de forme, de taille, de couleur et de tempérament (Figure 4). De l'analyse de la Figure 4, il ressort que la taille du corps $(87 \%)$ est plus utilisée pour distinguer les deux espèces de varan.

V. niloticus était sacré dans l'ethnie Batonu. Il cohabitait avec la population et n'était ni consommé, ni tué, ni vendu, ni sorti de son milieu.

Concernant la biologie de l'espèce, $66,7 \%$ des hommes et $76,7 \%$ des femmes ont dit savoir identifier le sexe des varans matures, difficile chez les immatures. La différenciation portait sur la présence d'hémipénis chez le mâle ainsi que la forme et la taille du corps. Les hommes se basaient sur le dimorphisme sexuel, les mâles étant plus grands de taille avec une tête moins effilée que chez les femelles. Par contre, les femmes se basaient surtout sur la forme du ventre qui est arrondie chez les femelles et plus prononcée quand elles portent des œufs.

\section{Evolution de la population des varans sacrés à Kandi \\ $V$. niloticus et $V$. exanthematicus} étaient moins rencontrés dans les champs et jachères (100\% des personnes interrogées) et les chasseurs n'avaient pas enregistré de capture. L'analyse de la perception de 63 chefs de ménage sur l'évolution de la population des varans sacrés révèle que l'effectif des varans sacrés toujours élevé (environ 100 individus) connaissait une baisse selon $61,9 \%$ des chefs de ménage dont $76,7 \%$ des femmes. Les causes avancées étaient la destruction de leur habitat $(57,14 \%$ des personnes interrogées), la perte du caractère sacré $(30,16 \%)$, les prélèvements clandestins $(50,79 \%)$, la consommation des œufs et des petits par les porcs $(68,25 \%)$, l'irrégularité des pluies $(7,94 \%)$ et la non-organisation de la cérémonie d' «ouverture de la voie des varans », à cause des mésententes nées des élections législatives. Les observations ont aussi permis de noter la présence des ectoparasites.

\section{Caractéristiques morphologiques}

Sur près d'une centaine de varans sacrés observés, 37 varans ont pu être capturés dont 6 immatures $(\mathrm{L}<85 \mathrm{~cm})$ et 31 matures $(\mathrm{L}>85 \mathrm{~cm})$.

\section{Données biométriques des varans}

La synthèse des données biométriques des varans sacrés est présentée dans le Tableau 2.

De l'analyse du Tableau 2, il ressort que :

La longueur de la queue des varans immatures est de 1,6 fois celle de leur museau-cloaque contre $1,5 \mathrm{chez}$ les varans matures. De même, le rapport de longueur totale des varans matures et immatures montre qu'à l'âge adulte, le varan pouvait quadrupler sa longueur et multiplier jusqu'à 67 fois son poids. L'indice de masse corporelle (IMC) est de 0,67 chez les varans immatures contre 1,20 chez les varans matures. Proportionnellement à la longueur du corps, les varans immatures sont donc légers par rapport aux varans matures. Enfin, les coefficients de variation élevés indiquent des groupes d'âge différents au sein de la population des varans sacrés qu'ils soient immature ou mature.

Covariance entre la longueur de la queue (LQ) et du museau-cloaque (LMC) puis entre la longueur totale ( $L$ ) et la masse corporelle (MC)

La Figure 5 illustre la corrélation entre les longueurs du Museau-cloaque (LMC) et de la queue (LQ) des varans sacrés.

La variation de LQ en fonction de LMC s'ajuste aux courbes de :

- tendance linéaire d'équation LQ = $1,19 \mathrm{LMC}+11,74$. tendance puissance d'équation $\mathrm{LQ}=$ $3,003 \mathrm{LMC}^{0,81}$, avec $\mathrm{R}^{2}=0,81$. 
De même, les coefficients de détermination des modèles logarithmique $\left(\mathrm{R}^{2}\right.$ $=0,74)$, polynomial $\left(\mathrm{R}^{2}=0,74\right)$ et exponentiel $\left(\mathrm{R}^{2}=0,74\right)$ sont élevés.

Les masses corporelles (MC) et les longueurs totales (L) des varans sacrés ont permis d'établir la covariance illustrée par la Figure 6.

La variation de $\mathrm{MC}$ en fonction de $\mathrm{L}$ s'ajuste à la courbe de tendance linéaire d'équation :

$\mathrm{MC}=0,03 \mathrm{~L}-1,42$, avec $\mathrm{R}^{2}=0,81$ (Figure 6).

Aussi, les modèles logarithmique $\left(\mathrm{R}^{2}=\right.$ $0,72)$, polynomial $\left(R^{2}=0,85\right)$, puissance $\left(R^{2}=\right.$ $0,96)$ et exponentiel $\left(\mathrm{R}^{2}=0,92\right)$ ont donné des coefficients élevés.

La valeur élevée obtenue pour les coefficients d'allométrie des cinq modèles traduit la forte corrélation existant entre les paramètres longueur du Museau-cloaque (LMC) et longueur de la queue (LQ) d'une part et masse corporelle (MC) et longueur totale (L) d'autre part.

\section{Importance des varans sacrés de Sam}

Les différents rôles joués par les varans selon les personnes interrogées sont résumés dans la Figure 7.

Il ressort de l'analyse de cette figure que sept différents rôles sont associés aux varans par les personnes interrogées. Cependant, les varans sacrés ne sont pas utilisés à des fins alimentaires et en médecine traditionnelle parce qu'ils ne peuvent être tués. De même, leur rôle économique n'est pas direct car ils ne peuvent être vendus.

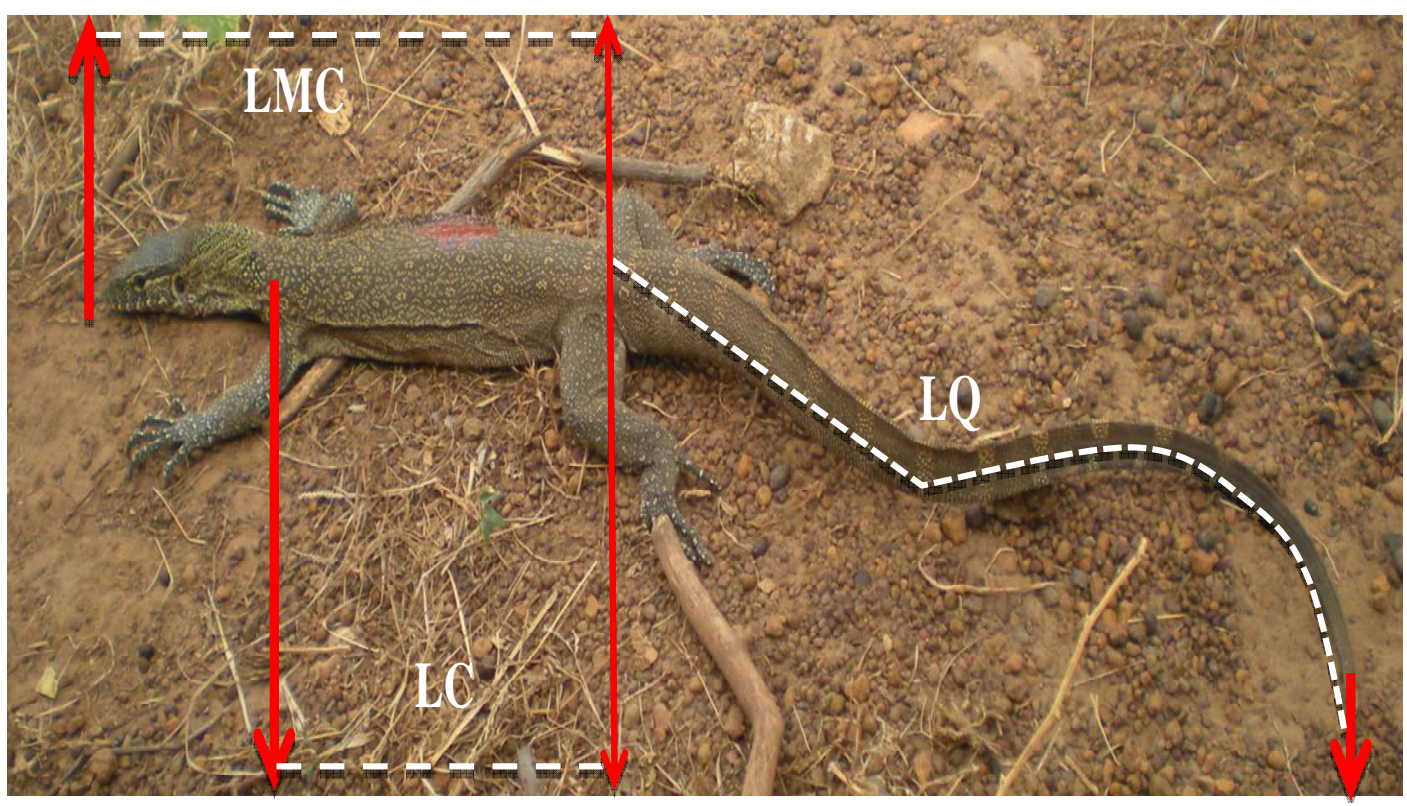

LMC : Longueur du Museau-Cloaque

LC : Longueur du corps

LQ : Longueur de la queue

Figure 2 : Illustration des mesures prises. 


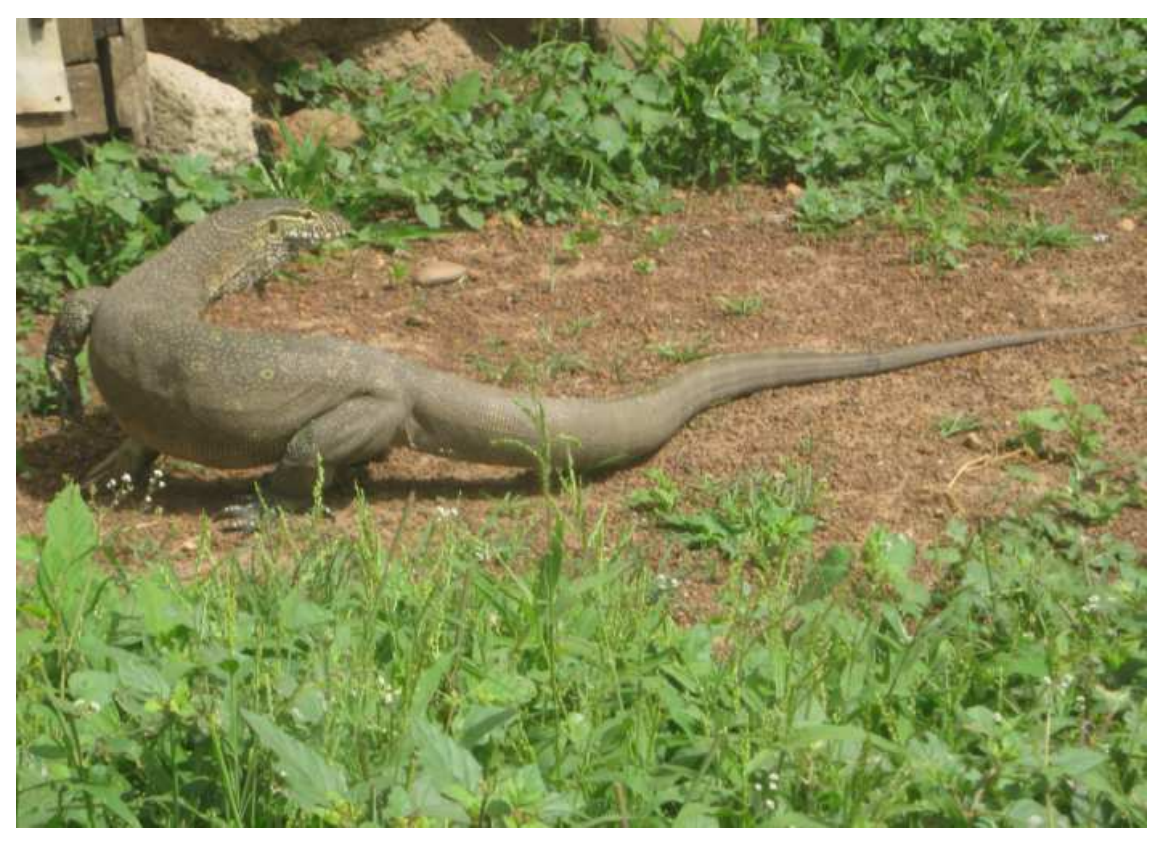

Figure 3 : Un varan du Nil sacré de Sam.

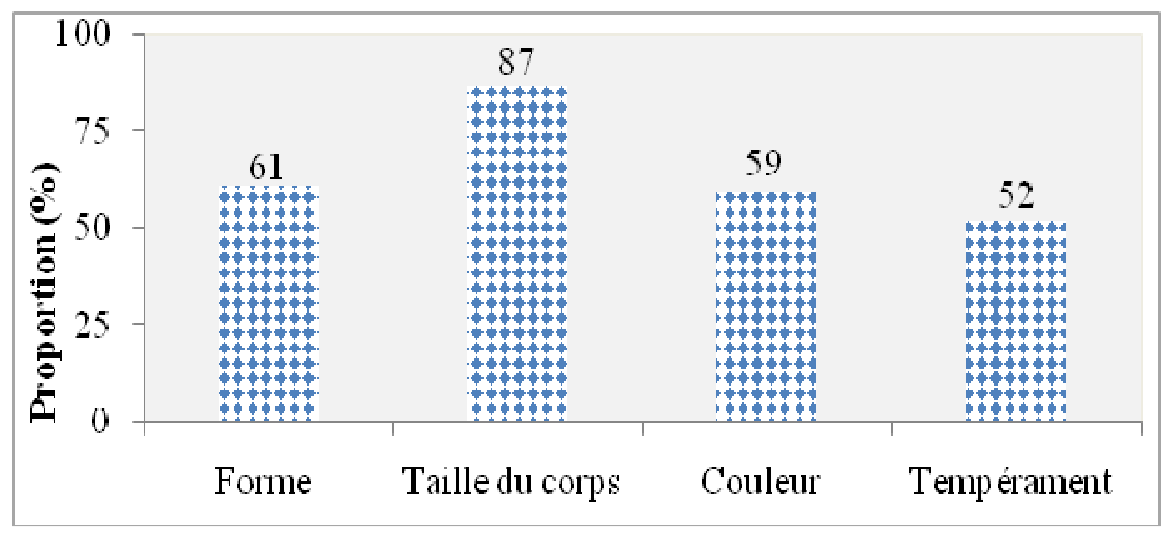

Figure 4 : Critères de discrimination du V. niloticus et $V$. exanthematicus 


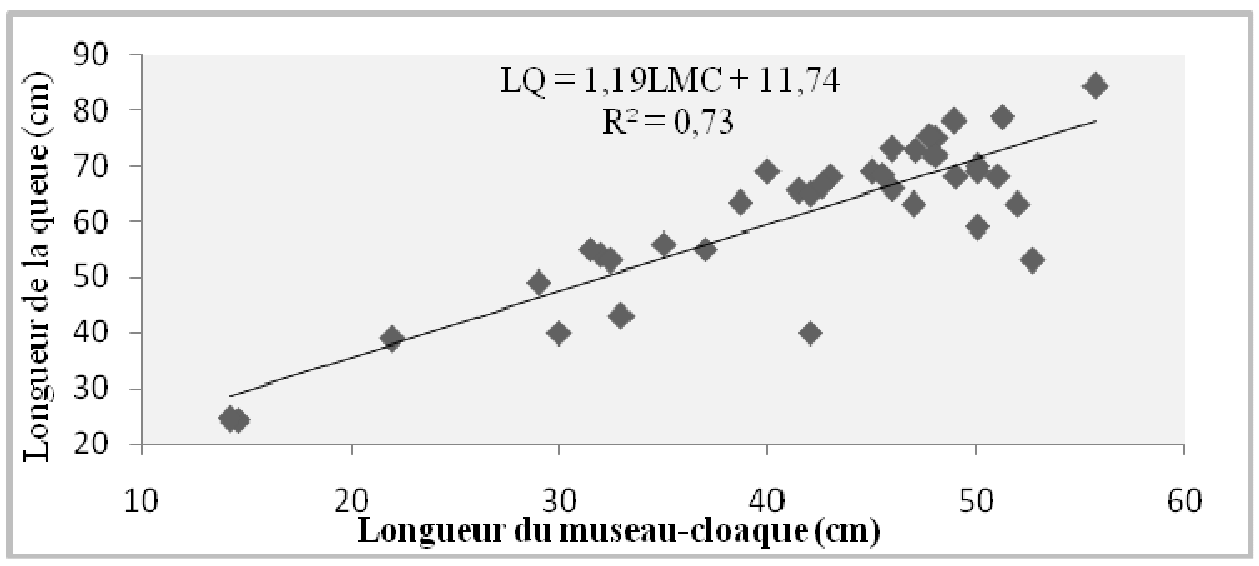

Figure 5: Droite de tendance linéaire de l'équation LQ = f(LMC).

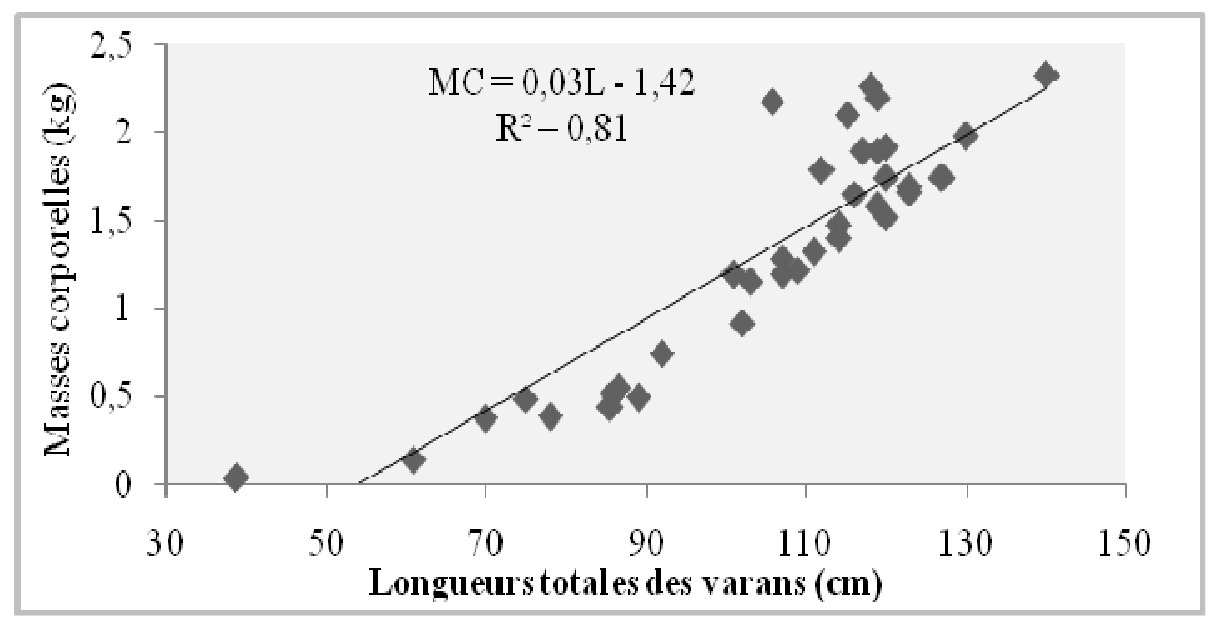

Figure 6: Droite de tendance linéaire de l'équation $\mathrm{MC}=\mathrm{f}(\mathrm{L})$.

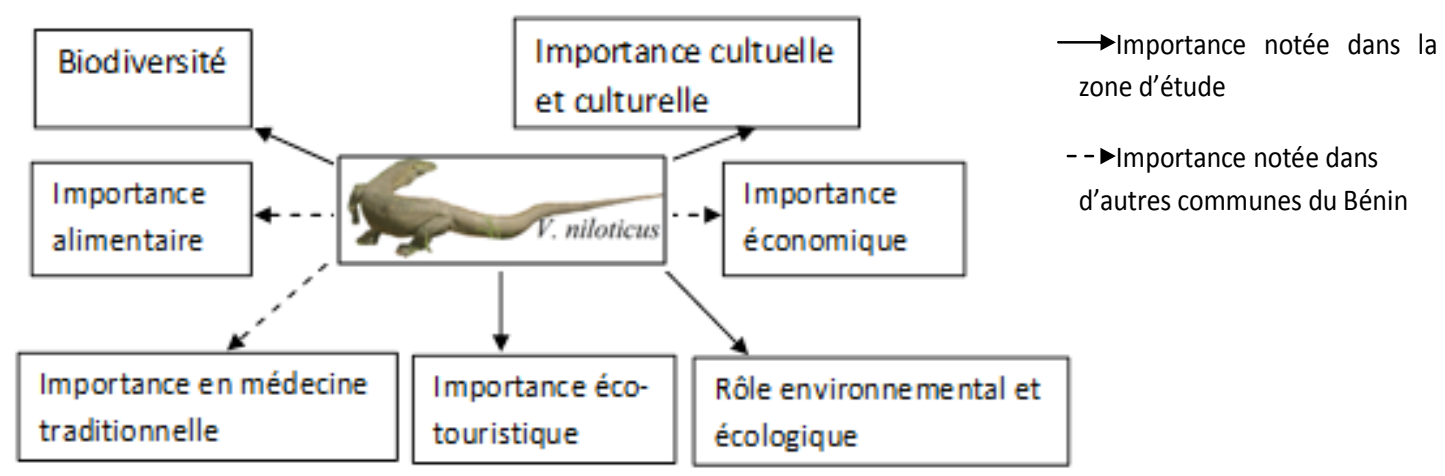

Figure 7: Importance du varan du Nil (Varanus niloticus). 
Tableau 1 : Description de V. niloticus et $V$. exanthematicus.

\begin{tabular}{lccccccc}
\hline & Taille & Museau & Tête & Cou & Queue & Couleur & Tempérament \\
\hline $\boldsymbol{V}$. niloticus & Grande & Allongé & Allongée & longs & Longue & Sombre & Agressif \\
$V$. exanthematicus & Moins grande & Court & Aplatie & Court & Courte & Plus claire & Calme \\
\hline
\end{tabular}

Tableau 2 : Données biométriques synoptiques des varans.

\begin{tabular}{|c|c|c|c|c|c|c|c|c|}
\hline \multirow{2}{*}{ Caractéristiques } & \multicolumn{4}{|c|}{ VARANS IMMATURES } & \multicolumn{4}{|c|}{ VARANS MATURES } \\
\hline & $\begin{array}{l}\text { LQ } \\
(\mathbf{c m})\end{array}$ & $\begin{array}{c}\text { LMC } \\
\text { (cm) }\end{array}$ & $\begin{array}{c}\mathbf{L} \\
(\mathbf{c m})\end{array}$ & $\begin{array}{c}\text { MC } \\
(\mathrm{g})\end{array}$ & $\begin{array}{l}\text { LQ } \\
(\mathrm{cm})\end{array}$ & $\begin{array}{c}\text { LMC } \\
(\mathbf{c m})\end{array}$ & $\begin{array}{c}\mathrm{L} \\
(\mathbf{c m})\end{array}$ & $\begin{array}{c}\text { MC } \\
(\mathrm{g})\end{array}$ \\
\hline Moyenne (CV) & $\begin{array}{c}38,8 \\
(32,9)\end{array}$ & $\begin{array}{c}24,1 \\
(35,7)\end{array}$ & $\begin{array}{c}60,3 \\
(29,2)\end{array}$ & $\begin{array}{l}244,2 \\
(79,7)\end{array}$ & $\begin{array}{c}65,3 \\
(15,3)\end{array}$ & $\begin{array}{c}44,7 \\
(14,5)\end{array}$ & $\begin{array}{c}111,3 \\
(12)\end{array}$ & $\begin{array}{c}1484 \\
(36,9)\end{array}$ \\
\hline Ecart-type & 12,8 & 8,6 & 17,6 & 194,5 & 10 & 6,5 & 13,4 & 547,7 \\
\hline Minimum & 24,4 & 14,2 & 38,7 & 35 & 40 & 31,5 & 85,5 & 436 \\
\hline Maximum & 56 & 35 & 78 & 485 & 84,3 & 55,7 & 140 & 2330 \\
\hline $\begin{array}{l}\mathrm{N} \text { (tailles de } \\
\text { l'échantillon) }\end{array}$ & 6 & 6 & 6 & 6 & 31 & 31 & 31 & 31 \\
\hline
\end{tabular}

\section{DISCUSSION}

Population et diversité des varans à Kandi

$V$. niloticus et $V$. exanthematicus les deux espèces signalées à Kandi, ont été mentionnées au nord-Bénin par Sinsin et al. (2008). Cette richesse spécifique de 2 est faible comparée au quatre de tout le continent africain. Varanus albigularis et Varanus griseus n'étaient pas signalés dans le milieu d'étude. De même, la sous-espèce Varanus niloticus ornatus, présente au sud Bénin, n'est pas signalée dans le milieu d'étude. Elle est reconnue comme étant de zone forestière (Leaché et al., 2006; Ullenbruch et al., 2010). Les caractéristiques biophysiques du milieu appartiennent par contre à l'intervalle de tolérance des espèces signalées. En effet, les deux espèces se retrouvent dans des formations de savanes, de forêts, de jachères et la présence de $V$. niloticus dans un milieu est particulièrement liée à la présence d'un point d'eau.

$V$. niloticus sacré de Sam est la seule population de varans sacrés signalés au Bénin. En effet, ni la revue de littérature ni les investigations n'ont permis de noter l'existence de varan sacré dans un autre milieu ou dans une autre communauté du Bénin. Par contre, le varan du désert (Varanus griseus) chez les Touaregs (Le Berre et Ramousse, 2003) et le varan du Nil et des savanes dans l'ethnie Séréres au Sénégal (Cissé, 1980) sont sacrés. De même, à Nioro au Mali, des varans cohabitent avec les populations comme à Sam (Kandi). Malgré la menace de la baisse drastique des varans des plusieurs milieux voire leur disparition (Sinsin et al., 2008), les prélèvements se poursuivent sans norme même dans certains sites sacrés (Ehinnou Koutchika et al., 2013). Le braconnage dans les aires protégées comme observé dans des pays comme le Cameroun (Sandjong Sani et al., 2013b) aggrave la menace. Le mécanisme de sacralisation des espèces doit être étudié, vu leur importance dans la conservation. En dehors de Sam (Kandi), les varans du Bénin sont très utilisés à des fins alimentaires, médicinales, etc. Ainsi, présents dans les milieux ouverts, les jachères et les champs, les varans des savanes, facilement capturés par les populations même par les femmes qui les ramassent, sont moins abondant que le varan du Nil. Mais, le varan du Nil doit sa survie à certains types d'habitats occupés. Dépendant 
de la présence de point d'eau, les zones marécageuses font partie de son habitat. Ces milieux difficiles d'accès à l'homme qui est la première menace de l'espèce, protège les populations de varans qui s'y trouvent. Cette protection n'est malheureusement pas définitive compte tenue des aménagements que l'homme apporte à ces milieux pour en faciliter l'exploitation et l'accès. Mittermeier (2008) fait remarquer que l'effectif des espèces de faune en général baisse à cause des prélèvements sans norme.

Les personnes interrogées ont souligné le dimorphisme sexuel comme Thompson and Pianka (2001). Mais, Ciliberti (2011) fait remarquer l'inexistence d'un dimorphisme sexuel évident portant sur les proportions du corps. Cependant, la présence d'hémipénis chez le mâle est confirmée par Schilliger (2005).

Les modifications de l'habitat sont reconnues par des biologistes de la conservation comme la principale cause de la perte de la biodiversité à travers le monde (Sala et al., 2000; Kaeslin et Williamson, 2010 ; Djego-Djossou et al., 2012). Dans la classification des menaces réalisée par Collins et Storfer (2003), les modifications de l'habitat, l'introduction d'espèces exotiques et la surexploitation figurent dans la classe I. Le porc (Sus scrofa Linné, 1758), introduit dans le milieu est cité sur la liste de l'IUCN parmi les 100 espèces les plus envahissantes au monde avec un impact direct sur les reptiles (Kirchner et Soubeyran, 2007). Il a déjà causé chez les oiseaux, la disparition du dodo (Raphus cucullatus) (Angst, 2011). Consommant les œufs de varans et les jeunes nouvellement éclos, il est urgent de contrôler son élevage ou au mieux l'exclure du milieu. D'autre part, la menace de la variabilité climatique figurant dans la classe II (Collins et Storfer, 2003) est pourtant une menace inquiétante en ce qu'il n'est pas facile d'y remédier (Gardner et al., 2007). Aussi, les pluies irrégulières font que, restés longtemps enfouis dans le sol, les œufs se gâtent ou les petits éclos meurent étouffés. Le faible nombre de varans juvéniles capturés en est une conséquence. En dehors de ces menaces, les varans sacrés de Sam sont confrontés à la perte du caractère sacré du fait de l'Islam et du Christianisme (Malhotra et al., 2001). Il faudra une reprise du culte des varans sacrés qui permettrait l'entretien du site sacré.

Les varans sont des espèces cannibales (Bayless et Luiselli, 2001). Les proies étant limitées à Sam, la consommation des juvéniles par les adultes est probable, favorisant un faible effectif des juvéniles. La perte du caractère sacré combinée aux nouvelles formes de construction empêchant l'installation des terriers pourrait à moyen terme et peut-être à court terme favoriser l'exclusion des varans sacrés.

\section{Covariance entre traits morphologiques chez les varans}

Les variables longueur du Museaucloaque (LMC), de la queue (LQ) et masse corporelle (MC) présentent de différences chez un même individu, entre individu de la population de varans sacrés et comparés à d'autres populations.

La variation de masse s'explique par la différence d'âge, les adultes pesant plus que les juvéniles et la différence des périodes de capture. Un varan est moins lourd en début de saison pluvieuse qu'en pleine saison pluvieuse à cause du ralentissement de croissance consécutif à la vie ralentie de saison sèche. Les mesures linéaires des varans sacrés notamment la LMC et la LQ sont significativement différentes $(p<0,05)$. Ainsi, la LMC était plus petite que la LQ. Cette différence est confirmée par les valeurs de 1,6 et $1,5 \mathrm{du}$ rapport entre la LQ et la LMC respectivement chez les individus immatures et matures qui sont du même ordre que la valeur de 1,55 obtenue avec les données des travaux de Schuett et al. (2009). Le passage à un mode de vie essentiellement terrestre n'a pas encore affecté significativement ce rapport.

L'équation LQ = 1,06 LMC + 21,48 de De Buffrénil et al. (1994), différente de celle trouvée, peut s'expliquer par la taille des échantillons. Concernant la fonction 
puissance, De Buffrénil et al. (1994) notaient que le coefficient d'allométrie b est toujours < 1 comme c'est le cas.

Quel que soit le modèle allométrique utilisé (linéaire, logarithmique, polynomial, puissance et exponentiel), les forts coefficients de détermination obtenus entre la LQ et LMC d'une part et entre la MC et la longueur totale (L) d'autre part traduisent leurs fortes corrélations confirmées par Collar et al. (2011) pour la LMC et LQ de plusieurs espèces de varans.

La longueur totale $(145,65 \mathrm{~cm})$, la LMC et la MC $(4,45 \mathrm{~kg})$ des varans matures des travaux de De Buffrénil et al. (1994) sont statistiquement plus élevées $(\mathrm{p}<0,05)$. De même, les mesures de la LMC et de la MC de Ciliberti et al. (2011) se situant respectivement entre $25,7 \mathrm{~cm}-67,2 \mathrm{~cm}$ et 320 g - 5660 g sont plus élevées que celles obtenues. En plus, le calcul des indices de masse corporelle (IMC) sur la base des valeurs moyennes des longueurs totales et masses corporelles révèle que l'IMC des travaux de De Buffrénil et al. (1994) est de 2,10 contre 1,6 pour les varans sacrés de Sam. Pour une même longueur totale, les varans de Sam sont donc moins lourds à cause d'une ressource alimentaire non suffisante. En effet, à la recherche de terres fertiles, les producteurs s'éloignent de plus en plus des habitations et s'y installent avec leur bétail en saison des pluies, période à laquelle les varans émergent alors que les larves des bouses de vache ne sont plus disponibles. La volaille également est amenée vers les fermes à cause des prélèvements importants. Privés de leur source d'alimentation, les varans ne font plus suffisamment de réserve avant d'entamer une nouvelle phase de quiescence justifiant leurs faibles poids. Chez les varans, les longueurs et masses corporelles influencent la performance de ponte. La conséquence du faible poids des varans sacrés serait la baisse de performance de ponte qu'il faudra vérifier pour des travaux futurs.

En outre, la difficulté dans l'identification du sexe des captures n'a pas permis de déterminer le sex ratio. Ainsi, une autre inquiétude se trouve dans la possibilité d'un faible nombre de sujets mâles. En effet, des prélèvements clandestins ont été signalés dans le stock sacré. Si les prélèvements ont ciblé des varans de grandes tailles, le risque est grand que des sujets mâles aient été les principales victimes étant donné que les mâles sont plus grands de taille.

Durant la saison des amours, les varans femelles s'accouplent avec plusieurs mâles pour garantir la fécondation de leurs œufs. Ces nombreux partenaires sexuels accroissent aussi la variabilité dans le matériel génétique de la descendance. Si des travaux ultérieurs indiquaient un faible nombre de mâles, les possibilités d'accouplement et la variabilité génétique seront réduites, favorisant ainsi la consanguinité et l'apparition de gènes létaux.

Les différences significatives entre les mesures corporelles des varans de Sam et des varans du lac Tchad suggèrent l'hypothèse d'une plasticité phénotypique chez les varans du Nil suivant le domaine géographique. Le Bénin étant divisé en districts phytogéographiques, une étude biométrique comparée des varans de ces zones s'avère nécessaire.

Au-delà du cultuel et culturel, les personnes interrogées ont reconnu l'importance des varans sacrés dans la protection des populations contre les morsures de reptiles comme les serpents qui entrent dans l'alimentation de ces varans (Ciliberti, 2011; Pianka, 2004). Les varans régulent aussi les dégâts dans les stocks de céréales car les rongeurs qui en sont responsables entrent dans leur alimentation (Sinsin et al., 2008; Savey, 2009).

Le varan sacré peut être une source de devises pour la commune à travers le développement de l'éco-tourisme comme le cas de Varanus komodoensis sur l'île de Komodo. Les impératifs tels que l'aménagement par l'administration forestière de refuges ou de site où les varans seront visibles aisément par les éventuels touristes doivent être réunis. Cet aménagement inclut le reboisement le long de la rivière de Sam, son curage et son enrichissement en espèces 
aquatiques afin que les chambres ne soient plus les premiers recours. La pérennisation de cet éco-tourisme doit passer par la mise en place de stratégies de conservation efficace et adaptée.

\section{Conclusion}

La survie des espèces notamment de la faune dépend de l'importance que les populations surtout locales leur accordent. Dans le village de Sam au Nord-est du Bénin, les varans du Nil (Varanus niloticus), l'une des deux espèces de varans présentes en dehors des varans de savane, revêtent un caractère sacré et cohabitent avec la population. Les captures marquées par un faible nombre des individus immatures (16\%) montrent l'urgence de prendre au sérieux les menaces identifiées car les varans de Sam offrent une grande chance de survie de l'espèce. En outre, si les faibles tailles comparatives obtenues suggèrent l'hypothèse d'une plasticité phénotypique suivant le domaine géographique, il faudra voir s'il ne s'agit pas d'un prélèvement poussé d'individus matures. La dégradation de la végétation environnante a mis les varans sacrés dans les conditions d'île écologique et qui pourraient avoir pour conséquence un fort taux de consanguinité. Les principales mesures à prendre sont d'une part l'aménagement du site des varans à travers le reboisement et l'installation de refuge et d'autre part la promotion de tourisme.

\section{REMERCIEMENTS}

Nous adressons nos sincères remerciements à la population de Sam (Kandi) pour avoir permis que cette étude puisse se faire sur les varans sacrés de leur village.

\section{REFERENCES}

AertsP, Van Damme R, Vanhooydonck B, Zaaf A, Herrel A. 2000. Lizard locomotion: how morphology meets ecology. Netherlands Journal of Zoology, 50: 261-277. DOI: $10.1163 / 156854200 \times 00126$
Angst D. 2011. «The end of the fat dodo? A new mass estimate for Raphus cucullatus ». Naturwissenschaften, 98: 233-236.DOI 10.1007/s00114-010-0759-7

Bayless MK, Luisselli L. 2001. A review of predation on African monitor lizards (Varanidae). Bulletin de la Société herpétologique de France, 97 : 19-26.

Bio Ouré R. 2011. Structure démographique et spatiale de Gerbilliscus kempi et Taterillusgracilis (Rodentia muridae) de la zone d'occupation contrôlée de la Réserve de Biosphère de la Pendjari au nord-ouest du Bénin. Mémoire d'Ingénieur Agronome. Université de Parakou, Bénin. p. 87.

Butler MA, King AA. 2004. Phylogenetic comparative analysis: a modeling approach for adaptive evolution. The American Naturalist, 164: 683-695.

Ciliberti A. 2011. Le varan du Nil (Varanus niloticus) indicateur de la pollution des zones humides d'Afrique sub-saharienne. Thèse de Doctorat. Université de Lyon, Lyon. p.158.

Ciliberti A, Berny P, Delignette-Muller M-L, De Buffrénil V. 2011. The Nile monitor (Varanus niloticus; Squamata: Varanidae) as a sentinel species for lead and cadmium contamination in sub-Saharan wetlands. Science of The Total Environment, 409 : 4735-4745. DOI: 10.1016/j.scitotenv. 2011.07.028 - Source: PubMed

Cissé M. 1980. Ecophysiologie comparée de deux varans en milieu sahélien (Sénégal). Thèse de doctorat ès sciences. Université de Nice, Nice. p. 339.

Collar DC, Schulte IIJA, Losos JB.2011. Evolution of extreme body size disparity in monitor lizards (Varanus). Evolution, 65(9): 2664-2680. DOI: 10.1111/j.15585646.2011.01335.x

Collar DC, Schulte II JA, O’Meara BC, Losos JB. 2010. Habitat use affects morphological diversification in dragon lizards (Agamidae). Journal of Evolutionary Biology, 23: 1033-1049. DOI: 10.1111/j.1420-9101.2010.01971.x 
Collins JP, Storfer A. 2003. Global amphibian declines: sorting the hypotheses. Diversity and Distributions, 9 : 89-98.

Daouda I-H A. 2002. Caractéristique structuro-pondérales, parasitisme par les cestodes et dynamique des populations de rongeurs dans la Presqu'Ile du Cap-Vert (Sénégal). Thèse de Doctorat du $3^{\text {ième }}$ Cycle de Biologie animale, Université Cheikh Anta Diop de Dakar. p. 143.

De Buffrénil V, Chabanet C, Castanet J. 1994. Données préliminaires sur la taille, la croissance et la longévité du varan du Nil (Varanus niloticus) dans la région du lac Tchad. Revue Canadienne de Zoologie,72: 262-273.DOI: 10.1139/z94-036

De Buffrénil V. 2004. L'exploitation et le commerce des varans africains : Le cas du varan du Nil (Varanus niloticus) au Tchad. Bulletin de la Société Zoologique de France, 129: 75-89.

Djego-Djossou S, Huynen MC, Djègo J, Sinsin B. 2012. Croyances Traditionnelles et Conservation du Colobe de Geoffroy, Colobus vellerosus (Geoffroy, 1834), dans la Forêt Sacrée de Kikélé, Bénin (Afrique de l'Ouest). African Primates, 7(2): 193202. http://hdl.handle.net/2268/167371

Djègo-Djossou S, Sinsin B. 2009. Distribution et statut de conservation du colobe de Geoffroy (Colobus vellerosus) au Bénin. International Journal of Biological and Chemical Sciences, 3(6): 1386-1397.

Dudley N, Higgins-Zogib L, Mansourian S. 2005. "Beyond Belief, Linking faiths and protected areas to support biodiversity conservation". A research report by WWF, Equilibrium and The Alliance of Religions and Conservation (ARC).

Ehinnou Koutchika RI, Agbani PO, Sinsin B. 2013. Influence des perturbations anthropiques sur la biodiversité des bois sacrés duCentre Bénin. International Journal of Biological and Chemical Sciences, 7(1): 306-318.

Gardner TA, Barlow J, Peres CA. 2007.Paradox, presumption and pitfalls in conservation biology: The importance of habitat change for amphibians and reptiles.
Biological Conservation, 138: 166-179. DOI: 10.1016/j.biocon.2007.04.017

INSAE. 2003. Troisième recensement général de la population et de l'habitat. Synthèses des résultats. Institut National de la Statistique et de l'Analyse Économique du Bénin (INSAE). Ministère du plan. Cotonou. Bénin. p. 34.

Kaeslin E, Williamson D. 2010. Forêts, populations et faune sauvage : Les enjeux d'un avenir commun. Unasylva, 61 : 3-10.

Kirchner F, Soubeyran Y. 2007. Espèces exotiques envahissantes : vers un état des lieuxglobal et un réseau d'action coordonné à l'échelle de tout l'outre-mer. 13eme ForumDes Gestionnaires Espèces Exotiques Envahissantes : Une Menace Majeure Pour LaBiodiversité Mnhn - Paris - Vendredi 16 Mars 2007. p. 5.

Kpéra GN, Mensah GA, Sinsin B. 2004.Utilisation des produits et sousproduits de crocodile en médecine traditionnelle au nord du Bénin. Bulletin de Recherche Agronomique du Bénin, $\mathrm{N}^{\circ} 44.12 \mathrm{p}$.

Leaché AD, Rödel M-O, Linkem CW, Diaz RE, Hillers A, Fujita MK. 2006. Biodiversity in a forest island: reptiles and amphibians of the West African Togo Hills. Amphibian and Reptile Conservation, 4(1): 22-45. DOI: 10.1514/journal.arc.0040018

Le Berre M, Ramousse R. 2003. Les enjeux de la conservation de la biodiversité en milieu saharien. Socioécologie et Conservation; Univ. Claude Bernard Lyon1; Villeurbanne France.

Luiselli L, Akani GC. 1999. Habitat of Snakes in the rainforest of Eket (Akwa-Ibom, State, south-eastern Nigeria) (Squamata/Serpentes). Herpetozoa, 11: 99-107.

Malhotra KC, Gokhale Y, Chatterjee S, Srivastava S. 2001. SCOPE Committee Publication. New Delhi and Bhopal, India: Indian National Science Academy and Indira Gandhi Rashtriya Manav Sangrahalaya. 
Mensah GA, Akpona HA, Guidigbi EAT, Ogouma EE, Pomalegni SCB, Toudonou CAS, Yolou DA. 2006. Inventaire des mammifères rongeurs et des reptiles dans la réserve de Biosphère de la Pendjari. Rapport technique final. GTZ. Bénin. p. 140.

Mittermeier RA. 2008. La Menace d'Extinction s'Intensifie sur les Espèces les plus Proches de l'Homme. New Press edition.

Openshaw GH, Keogh JS. 2014. Head shape evolution in monitor lizards (Varanus): Interactions between extreme size disparity, phylogeny and ecology. Journal of Evolutionary Biology, 27: 363-373. DOI: $10.1111 /$ jeb.12299

Pianka ER. 2004. Varanus olivaceus. In Varanoid Lizards of the World, Pianka ER, King DR, King RA (eds). Indiana University Press: Bloomington, EUA; 220-224.

Pianka ER, King DR. 2004. Varanoid Lizards of the World., Indiana University Press: Bloomington.

Sala OE, Chapin FSI, Armesto JJ, Berlow E, Bloomfield J, Dirzo R, Huber-Sanwald E, Huenneke LF, Jackson RB, Kinzig A, Leemans R, Lodge DM, Mooney HA, Oesterheld M, Poff NL, Sykes MT, Walker BH, Walker M, Wall DH. 2000. Global biodiversity scenarios for the year 2100. Science, 287(5459): 1770-1774. DOI:10.1126/science.287.5459.1770

Sandjong Sani RC, Ntoupka M, Ibrahima A, TVroumsia. 2013a. Etude écologique du Parc National de Mozogo-Gokoro (Cameroun): prospections préliminaires de la flore ligneuse et du sol pour sa conservation et son aménagement. International Journal of Biological and Chemical Sciences, 7(6): 2434-2449.

Sandjong Sani RC, Ntoupka M, Ibrahima A, T Vroumsia. 2013. Essai d'analyses de la conception paysanne de l'évolution, de la gestion et de l'utilité du Parc National de Mozogo-Gokoro (Cameroun) en vue de son aménagement. International Journal of Biological and Chemical Sciences, 7(6): 2490-2503.

Savey C. 2009.Les affections des lézards liées aux conditions de captivité. Thèse de Doctorat Vétérinaire, Ecole Nationale Vétérinaire d'Alford. p. 136.

Schilliger L. 2005. Déterminisme et détermination du sexe chez les Reptiles. La Nachronique, 16: 21-24.

Schuett GW, Reiserer RS, Earley RL. 2009. The evolution of bipedal postures in varanoid lizards. Biological Journal of the Linnean Society, 97: 652-663. DOI: 10.1111/j.1095-8312.2009.01227.x

Sinsin B, Zannou A, Djossa B, Kpéra GN, Tchibozo N. 2008. Biologie des principaux spécimens de la convention Internationale sur le commerce des espèces en Danger (CITES) au Bénin : cas des varans, tortues, caméléons et scorpions. LEA/FSA/UAC et PGFTR/MEPN. p. 41.

Smart R, Whiting MJ, Twine W. 2005. Lizards and landscapes: integrating field surveys and interviews to assess the impact of human disturbance on lizard assemblages and selected reptiles in a savanna in South Africa. Biological Conservation, 122: 23-31. DOI: 10.1016/j.biocon. 2004.06.016

Thompson GG, Pianka ER. 2001. Allometry of Clutch and Neonate Sizes in Monitor Lizards (Varanidae: Varanus). Copeia, 2: 443-458.

Ullenbruch K, Grell O,Böhme W. 2010. Reptiles from southern Benin, West Africa, with the description of a new Hemidactylus (Gekkonidae), and a country-wide checklist. Bonn Zoological Bulletin, 57(1):31-54. 